\title{
Impacto ambiental en suelos sometidos a explosivos en San Luis, Tolima (Colombia)
}

\author{
Soil Environmental Assessment by Explosives in San Luis, Tolima (Colombia)
}
Impacto ambiental em solos submetidos a explosivos em San Luis, Tolima (Colômbia)

\author{
Rosalina González Forero* | Gabriel Darío Romero Guerra ${ }^{\mathrm{b}}$ | Miguel Ángel Echeverría Vargas \\ a https://orcid.org/0000-0002-7948-9085 Universidad de La Salle, Bogotá, Colombia \\ ${ }^{\text {b }}$ https://orcid.org/0000-0002-3008-7456 Policía Nacional de Colombia, Bogotá, Colombia \\ chttps://orcid.org/0000-0003-09|4-5559 Policía Nacional de Colombia, Bogotá, Colombia
}

- Fecha de recepción: 2020-06-02

- Fecha concepto de evaluación: 2020-08-13

- Fecha de aprobación: 2020-08-2I https://doi.org//0.22335/rlct.v12i3.1254
Para citar este artículo / To reference this article / Para citar este artigo: González Forero, R., Romero Guerra, G. D., \& Echeverría Vargas, M. A. (2020). Impacto ambiental en suelos sometidos a explosivos en San Luis, Tolima (Colombia). Revista Logos Ciencia \& Tecnología, I2(3), 47-56. https://doi.org/I0.22335/rlct.v I 2i3.I 254

\section{RESUMEN}

El proyecto realizado identificó la alteración que pueden causar los explosivos en el suelo luego de realizar detonaciones controladas con pentolita, pentrita y $\mathrm{C} 4$ en terrenos identificados del municipio de San Luis, Tolima. Lo anterior sirve de base para proponer un procedimiento que apoye la lucha contra la contaminación ambiental. Esto se llevó a cabo utilizando un diseño metodológico estructurado con un tipo de estudio descriptivo-experimental con enfoque mixto. Los resultados obtenidos se soportaron en un trabajo de campo que siguió los protocolos que en materia de suelos propone el Instituto Geográfico Agustín Codazzi (IGAC), así como de métodos estándar de laboratorio del Centro Tecnológico de Ambiente y Sostenibilidad de la Universidad de la Salle, con el fin de determinar las propiedades del recurso suelo, midiendo las variaciones en sus componentes antes y después de las detonaciones de manera controlada. Las propiedades medidas fueron: materia orgánica, nitrógeno amoniacal y nitratos. Los resultados obtenidos en este trabajo permitieron identificar el impacto ambiental en el suelo debido a la variación de las propiedades fisicoquímicas en este tales como materia orgánica y especies con nitrógeno en forma de nitratos y nitrógeno amoniacal.

Palabras clave: pentrita, pentolita, C4, recurso suelo, explosivos 


\begin{abstract}
The project undertaken identified the alteration to the land that explosives can cause after carrying out controlled detonations with pentolite, pentrite and C4 in identified lands in the municipality of San Luis, Tolima. The above serves as the basis for proposing a procedure that supports the fight against environmental pollution. This was carried out using a structured methodological design with a descriptive-experimental type of study which followed a mixed approach. The results obtained were supported by field work that followed the protocols proposed by the Agustín Codazzi Geographical Institute (IGAC), as well as the standard laboratory methods proposed by the Technology Centre for Environment and Sustainability from the La Salle University, in order to determine the properties of the land resources, measuring the variations in its components before and after detonations in a controlled manner. The properties measured were: organic matter, ammonia nitrogen, and nitrates. The results obtained in this project helped identify the environmental impact on the land due to the variation of the physicochemical properties in it, such as organic matter and species with nitrogen in the form of nitrates and ammoniacal nitrogen.
\end{abstract}

Keywords: pentrite, pentolite, C4, soil resource, explosives

\title{
RESUMO
}

O projeto realizado identificou a alteração possivelmente causada pelos explosivos no solo após a realização de detonações controladas com pentolita, pentrita e $\mathrm{C} 4 \mathrm{em}$ terrenos identificados do município de San Luis, Tolima. O supracitado serve de base para sugerir um procedimento que suporte a luta contra a contaminação ambiental. Isto se realizou utilizando um desenho metodológico estruturado com um tipo de estudo descritivo-experimental com uma abordagem mista. Os resultados obtidos foram alavancados por um trabalho de campo que seguiu os protocolos que, em matéria de solos, propõe o Instituto Geográfico Agustín Codazzi (IGAC), assim como de métodos padrão de laboratório do Centro Tecnológico de Ambiente e Sustentabilidade da Universidade de La Salle, com o objeto de determinar as propriedades do recurso solo, medindo as variações em seus componentes antes e depois das detonações de maneira controlada. As propriedades medidas foram: matéria orgânica, nitrogênio amoniacal e nitratos. Os resultados obtidos com este trabalho permitiram identificar o impacto ambiental no solo devido à variação de suas propriedades físico-químicas, tais como matéria orgânica e espécies com nitrogênio em forma de nitratos e nitrogênio amoniacal.

Palavras-chave: pentrita, pentolito, C4, recurso solo, explosivos

Actualmente, la contaminación ambiental se ha convertido en un tema que ha centrado la atención de los gobiernos del mundo, debido a la afectación directa en recursos naturales tales como agua, aire y contaminación de los suelos (Programa de las Naciones Unidas para el Desarrollo [PNUD], 2020). En particular, esta problemática en suelos ha sido poco explorada y el manejo inadecuado de residuos explosivos en este recurso, así como el destino ambiental de estos no se ha estudiado en el país (González \& Ortiz, 2020), lo que puede contribuir a generar efectos perjudiciales para la salud humana. Esta problemática ha generado alta preocupación en las autoridades y entes académicos, en particular en la Escuela de Investigación Criminal y de la Universidad de La Salle, quienes han adelantado labores de investigación y trabajo coordinado en la búsqueda de alternativas de control de la contaminación (González \& Murillo, 2016).
Durante décadas, Colombia ha sufrido los embates de un conflicto armado que ha afectado los ecosistemas del país. Una de las razones es el uso de sustancias explosivas en los territorios en los que hacen presencia los grupos armados organizados al margen de la ley, pues esto ha promovido los controles de la Fuerza Pública de acuerdo con la prohibición del uso de sustancias explosivas tales como la pentrita, la pentolita y el C4 ( ciclonita + plastificante), entre otras. Estos controles se han ejercido por medio de procedimientos de incautación y desactivación de las sustancias explosivas en forma de explosión, combustión o disolución (Villate \& Lozano, 2008).

Con base en lo anterior, se evidencia que a pesar de la prioridad de labores de desminado dentro de los acuerdos de paz pactados entre el Gobierno colombiano y las FARC-EP, aún existen zonas afectadas por explosivos en el 
país. Además, no se ha realizado una medición del impacto ambiental ocasionado en esos territorios (suelos, fuentes hídricas, biodiversidad), ni mucho menos se ha responsabilizado administrativa o penalmente a quienes participaron de forma activa en generar contaminación ambiental durante el conflicto armado (González \& Ortiz, 2020).

La presente investigación busca identificar cuáles pueden ser los posibles efectos generados en el suelo a partir de las detonaciones de explosivos, así como aportar información importante para las futuras investigaciones en casos relacionados con la contaminación ambiental. De este modo, se continúa con la discusión de si estas actividades y procesos que se realizan con frecuencia en nuestro país aportan directa $o$ indirectamente al deterioro ambiental en los territorios.

En Colombia se han realizado pocas investigaciones que sigan la línea investigativa de suelos para determinar el impacto ambiental que las sustancias explosivas puedan causar en el medio, quizás por falta de interés, falta de medios o la heterogeneidad de estos (González \& Ortiz, 2020; González \& Murillo, 2016; González, Ortiz \& Erazo, 2017). Ahora bien, estas investigaciones se han enfocado dentro del tipo descriptivo documental, más que experimental, por ende, para una institución garante de derechos y libertades públicas como lo es la Policía Nacional de Colombia es de vital importancia conocer sobre los medios que conllevan a la contaminación ambiental, a fin de innovar en el desarrollo investigativo de los factores asociados que, posteriormente, permitirán establecer criterios y nuevas metodologías para el abordaje integral del fenómeno.

Cabe esperar que los resultados obtenidos aporten nuevos datos sobre los impactos que generan las detonaciones de los explosivos usados por grupos al margen de la ley en suelos colombianos. Además, es evidente que el tema de estudio es amplio y genera cada vez más interrogantes $y$, por ende, mayor interés por explorar nuevas líneas investigativas que abarquen el tema de estudio, promoviendo siempre la búsqueda de mejores condiciones de vida para que las generaciones venideras logren entender que la única manera de asegurar el futuro y la vida se encuentra en la conservación del medioambiente en el que vivimos.

\section{Metodología}

La presente investigación se realizó con base en un estudio de tipo descriptivo-experimental con enfoque multimodal o mixto (Teddlie \& Tashakkori, 2003). En este se buscó identificar el impacto ambiental de sustancias explosivas C4, PENT y pentolita en suelo el cual está ligado directamente al enfoque cualitativo (comprensión de la singularidad del terreno) y cuantitativo (recopilación de información concreta).Teniendo en cuenta el desarrollo del enfoque investigativo mixto de la presente investigación (Hernández, Fernández \& Baptista, 2010), fue necesario vincular datos cuantitativos (datos estadísticos, porcentuales) y cualitativos por medio de la observación (condiciones del terreno y afectación de este) basados en los instrumentos. Lo anterior con el fin de responder al planteamiento del problema, por el cual se logró determinar el impacto que se genera en el medio ambiente por medio de la comparación entre las características fisicoquímicas (materia orgánica y nitrógeno en forma de nitratos y nitrógeno amoniacal) posterior a la detonación de explosivos en suelos del municipio del San Luis, Tolima. Cabe anotar que no existe normatividad colombiana en el tema de contaminación de suelos y menos con sustancias explosivas.

Las pruebas de campo se realizaron en terrenos identificados y debidamente controlados en el municipio de San Luis, Tolima, con el apoyo del personal de estudiantes de la Técnica Profesional en Explosivos de la Escuela de Investigación Criminal. Es importante anotar que no es posible utilizar cualquier tipo de terreno, en razón a la peligrosidad que se deriva del uso de explosivos $y$, por ende, el lugar tuvo que ser muy controlado, lo que limita la posibilidad de utilizar diferentes tipos de suelos en lugares diversos. El suelo de la zona de estudio es de litología cuaternaria de depósitos fluviovolcánicos, con paisaje de piedemonte y un clima tropical lluvioso de bosque (Instituto Geográfico Agustín Codazzi [IGAC], 1997). Se trabajó con una población definida, es decir, finita, en un área aproximada de $500 \mathrm{~m}^{2}$ en donde fueron recolectadas las muestras de suelo natural y aquellas provenientes de las detonaciones con los distintos explosivos, las cuales se tomaron después de cada detonación. Se realizaron tres detonaciones para cada sustancia explosiva (pentolita, pentrita, C4) en suelo a nivel superficial (entre $0-10 \mathrm{~cm}$ de profundidad); de ellas se tomaron tres submuestras teniendo el radio de afectación del suelo para, posteriormente, mezclarlas en aras de obtener una muestra compuesta representativa de entre 500 y $700 \mathrm{~g}$ de cada material explosivo; cabe anotar que la selección del peso y las cargas utilizadas obedeció a la presentación comercial de estas (véase la tabla I). Posteriormente, las muestras fueron llevadas al laboratorio en el Centro Tecnológico de Ambiente y Sostenibilidad (CTAS) de la Universidad de La 
Salle para su análisis, teniendo en cuenta los protocolos de embalaje, transporte y almacenamiento del Instituto Geográfico Agustín Codazzi (IGAC, 2017).

Los datos fueron recolectados mediante el instrumento diario de campo para la toma de muestras, y los análisis de laboratorio se recolectaron mediante una bitácora de laboratorio, con un esquema tipo rejilla (véase la tabla 2) (Hernández, Fernández \& Baptista, 20I4).

Para el análisis fisicoquímico en el laboratorio (tabla 3) se utilizaron las siguientes metodologías para el estudio del suelo, útiles en el propósito de identificar parámetros que varían o pueden afectarse después de la detonación de un explosivo.

\section{Resultados}

\section{Observación en campo luego de la detonación de C4}

Las tres detonaciones con el explosivo C4 causaron daño morfológico visible en el suelo, lo cual provocó una gran cantidad de material particulado y dejó una marca de $6 \mathrm{~m}$ de

Tabla I. Muestras de suelo recolectadas en el estudio (suelo natural y suelo después de las detonaciones con cada explosivo)

\begin{tabular}{c|l|c} 
Número de muestra & \multicolumn{1}{c|}{ Clase de muestra } & Peso en seco \\
\hline $\mathrm{I}$ & Suelo natural & $500 \mathrm{~g}$ \\
\hline 2 & Suelo con pentrita (cordón detonante $94 \mathrm{~cm}, 12 \mathrm{~g}$ cada m) & $700 \mathrm{~g}$ \\
\hline 3 & Suelo con pentolita I $125 \mathrm{~g}$ & $700 \mathrm{~g}$ \\
\hline 4 & Suelo con pentolita $2,250 \mathrm{~g}$ & $700 \mathrm{~g}$ \\
\hline 5 & Suelo con C-4 barras de I,25 lb & $700 \mathrm{~g}$ \\
\hline
\end{tabular}

Tabla 2. Procedimiento de campo para la recolección de muestras de suelo preexplosión y posexplosión

\section{Diario de campo}

Fecha: 6/1 I/2019

\section{Lugar: San Luis, Tolima}

\section{Tema: Práctica de recolección de evidencia de suelo}

Objetivo: recolección de muestras de suelo preexplosión y posexplosión para su análisis

\section{Ejes temáticos}

Ia. Características

Ib. Objetivo del trabajo

Ic. Desarrollo de la salida

2. Equipos a utilizar

3. Atención de situaciones imprevistas

4a. Planeación.

4b. Trabajo con el grupo.
Elementos de seguridad (guantes, tapabocas, tapaoídos), chaleco antibalas, palas, recipientes herméticos, cordón detonante, detonadores, C4, pentrita, pentolita, sonómetro, anemómetro.

Grupo altamente capacitado en manejo de explosivos.

Calibración de los instrumentos, elaboración del plan de marcha, especificando ruta, personal y vehículos a utilizar, solicitud acompañamiento de grupo altamente capacitado en el manejo de explosivos, preparación de los elementos, selección del terreno para las detonaciones, toma de muestras al sue-

Con el fin de tomar muestras en suelo, las cuales se llevarán a laboratorio junto con las muestras de suelo natural para determinar materia orgánica, nitrógeno amoniacal, nitritos y nitratos. lo preexplosión, preparación de los explosivos por parte del grupo de explosivos, detonación controlada de los explosivos por personal capacitado, siguiendo medidas de seguridad, recolección en bolsas de polietileno de I kg de capacidad, embalaje y rotulado de muestras por parte de los investigadores, toma material fotográfico y de video.
Se observa un suelo color café oscuro, medio húmedo, con presencia de vegetación y fauna (insectos).

Activación de la carga de pentolita; luego de activarse se realiza la observación en terreno, gran poder de destrucción, considerable material particulado; luego de asegurar el terreno se recolecta la muestra, el suelo tiene una coloración negruzca, no hay presencia de vida al ojo humano.

Activación de la carga con pentrita; en comparación con la pentolita su explosión es menos fuerte, menos cantidad de material particulado en el ambientes, gran capacidad de destrucción, tierra color negro, no hay presencia de vida a simple vista.

Activación C4; bajo la percepción de los sentidos la detonación es más fuerte que la pentrita, similar a la detonación del explosivo pentolita, deja más deflagración de humo que las anteriores, tierra color negro, vegetación quemada 
Tabla 3. Métodos de laboratorio utilizados para la medición de parámetros

\begin{tabular}{|c|c|c|}
\hline Ensayo & Método & Fuente \\
\hline Nitrógeno amoniacal & Salicilato & Hach Company (200I) \\
\hline Nitratos & Dimetilfenol & Hach Company (200I) \\
\hline Materia orgánica & Walkie-Black & Okalebo, Gathua y Woomer (2002) \\
\hline
\end{tabular}

largo por $3 \mathrm{~m}$ de ancho, así como una profundidad de aproximadamente $0,5 \mathrm{~cm}$ y una deflagración de humo color gris oscuro que tardó, aproximadamente, tres minutos en dispersarse en su totalidad.

La acción del explosivo provocó incremento aproximado de la temperatura del suelo entre los 60 y $70^{\circ} \mathrm{C}$, lo cual pudo apreciarse al tacto. La fauna y flora presentes en el terreno removido por el explosivo resultaron completamente carbonizados, por consiguiente, el suelo y su alrededor tomó un color gris oscuro (véase la figura I).

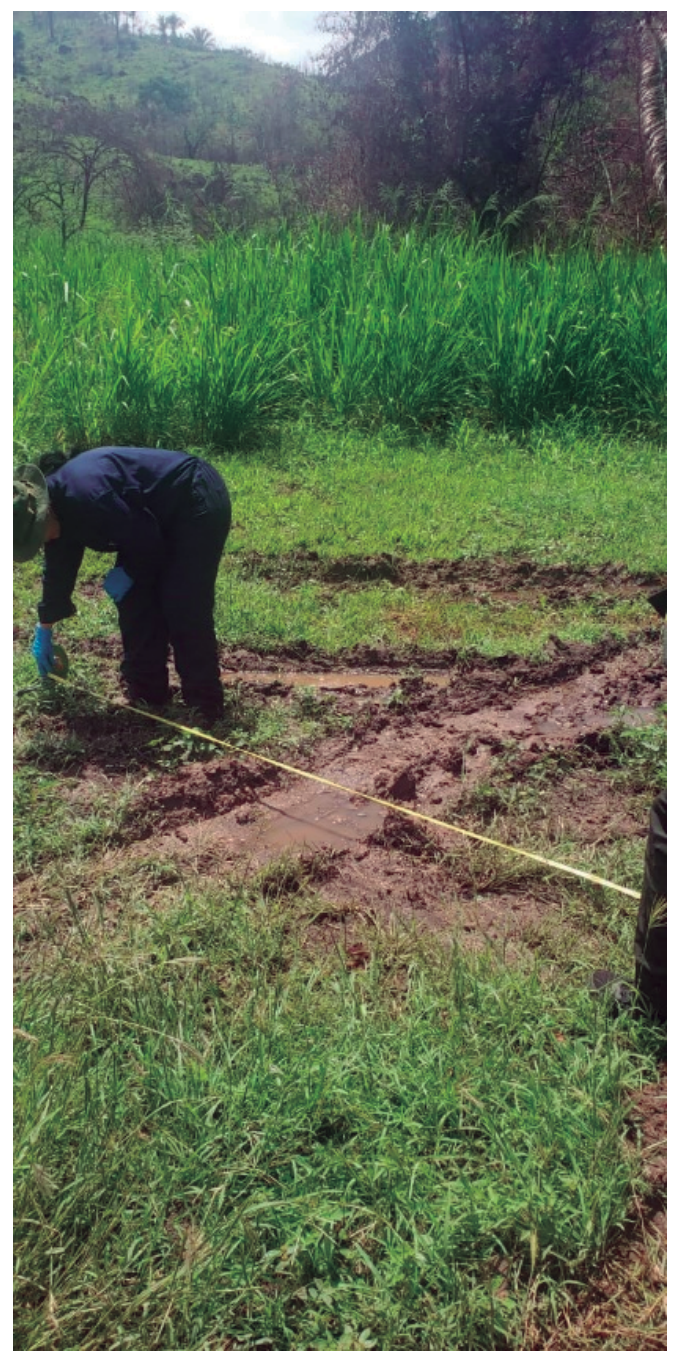

Figura I. Suelo después de la detonación carga C4.
Luego de exponer el terreno a las detonaciones del explosivo $\mathrm{C} 4$, el contenido de materia orgánica se redujo en un $89,21 \%$, al emplear $1,25 \mathrm{lb}$ del explosivo, en comparación con el contenido del suelo natural (véase la tabla 4); el efecto sobre este parámetro es devastador debido a las características del explosivo, pues su efecto es de impacto dirigido. Esta variación tan grande se debe a que toda la capa orgánica del punto específico de detonación fue removida por la reacción.

La materia orgánica influye en la activación y la proliferación microbiana, por ende, también facilita la captación de los cationes, el agua y el oxígeno necesarios para un mejor desarrollo y nutrición vegetal. El proceso de degradación de materia orgánica es inducido por la acción directa del hombre (acondicionamiento artefacto explosivo), lo cual implica disminución de la fertilidad (Rojas, 2015).

Tabla 4. Cantidad de materia orgánica en suelo natural y en suelo expuesto a detonación $\mathrm{C} 4$

\begin{tabular}{cccc} 
Muestra de suelo & $\begin{array}{c}\text { Materia } \\
\text { orgánica }(\%)\end{array}$ & $\begin{array}{c}\text { Diferen- } \\
\text { cia }(\%)\end{array}$ & $\begin{array}{c}\text { Diferencia } \\
\text { porcentual }\end{array}$ \\
\hline Natural & 2,24 & 2 & 89,28 \\
\hline Detonado con carga C4 & 0,24 & 2 & \\
\hline
\end{tabular}

Se aprecia un ligero aumento en el contenido de nitratos para la muestra expuesta a la detonación con $\mathrm{C} 4$ con relación a la concentración inicial (véase la tabla 5). Se infiere que para este tipo de explosivo los nitratos mantienen su concentración sin verse afectados significativamente por las reacciones ocurridas en la explosión. Lo anterior se debe a que la reacción de descomposición de los nitratos genera nitritos en los explosivos (División Técnica de Armamentos Terrestres, 1984).

Tabla 5. Cantidad de nitratos en suelo natural y en suelo expuesto a detonación C4

\begin{tabular}{cccc} 
Muestra de suelo & $\begin{array}{c}\text { Nitratos } \\
\mathbf{m g} / \mathrm{L}\end{array}$ & $\begin{array}{c}\text { Diferencia } \\
\mathrm{mg} / \mathrm{L}\end{array}$ & $\begin{array}{c}\text { Diferencia } \\
\text { porcentual }\end{array}$ \\
\hline Natural & 0,2 & 0,1 & 50 \\
\hline Detonado con C4 & 0,3 & 0,1 & \\
\hline
\end{tabular}


El contenido de $\mathrm{N}$ amoniacal registrado en el momento de la explosión aumentó un $290 \%$ con relación a la cantidad de amonio presente en la muestra de suelo natural (véase la tabla 6). Se aprecia una evidente alteración en la composición de la muestra de suelo, debido al aporte de residuos del explosivo, ya que dentro de su composición se encuentra presente el nitrato amónico (Villate \& Lozano, 2008). Cabe anotar que con la cantidad de explosivo utilizado no se altera la muestra a niveles tóxicos de la sustancia para el suelo (cantidad normal de I a $5 \mathrm{mg} / \mathrm{L}$ ); sin embargo, una exposición constante o de mayor volumen de explosivo podría alterar los valores normales (Antón \& Lizaso, 200I).

Tabla 6. Cantidad de $\mathrm{N}$ amoniacal en suelo natural $\mathrm{y}$ en suelo expuesto a detonación $\mathrm{C} 4$

\begin{tabular}{cccc} 
Muestra de suelo & $\begin{array}{c}\text { N Amoniacal } \\
\text { mg/L }\end{array}$ & $\begin{array}{c}\text { Diferencia } \\
\text { mg/L }\end{array}$ & $\begin{array}{c}\text { Diferencia } \\
\text { porcentual }\end{array}$ \\
\hline Natural & $\mathrm{I}$ & 2,9 & 290 \\
\hline Detonado con C4 & 3,9 & & \\
\hline
\end{tabular}

\section{Observación en campo luego de la detonación de PENT}

En cada una de las tres detonaciones se utilizaron $1 \mathrm{I}, 28 \mathrm{~g}$ (94 cm de cordón detonante) de pentrita (PENT), se generó un daño visible en el suelo de, aproximadamente, $70 \mathrm{~cm}$ de ancho por $5 \mathrm{~m}$ de largo y $\mathrm{I} \mathrm{cm}$ de profundidad, la combustión del explosivo provocó la compactación del material orgánico de la capa superficial (horizonte 0 ) con las raíces de la vegetación quemadas, al igual que organismos vivos tales como insectos (véase la figura 2). Luego de la detonación, la cantidad de material particulado y humo visible en el aire tardó aproximadamente 45 s para su dispersión total.

La materia orgánica aumenta si se tiene en cuenta que el explosivo PENT se deposita sobre el terreno en forma de cordón detonante; luego de efectuarse su detonación dejó en el terreno residuos de carbonos que aumentaron la materia orgánica en un $\mathbf{9 9 , 7 7 \%}$ (véase la tabla 7 ). Toda vez que el componente explosivo tiene una estructura alifática con alto contenido de carbonos (García, 2013), esto posiblemente ocasionó la variación de los valores en la muestra.

El contenido de nitratos en el suelo detonado con PENT aumentó $1200 \%$ en comparación con la cantidad de este nutriente en el suelo natural (véase la tabla 8 ). Se evidencia que, para la muestra sometida a este tipo de explosivo,

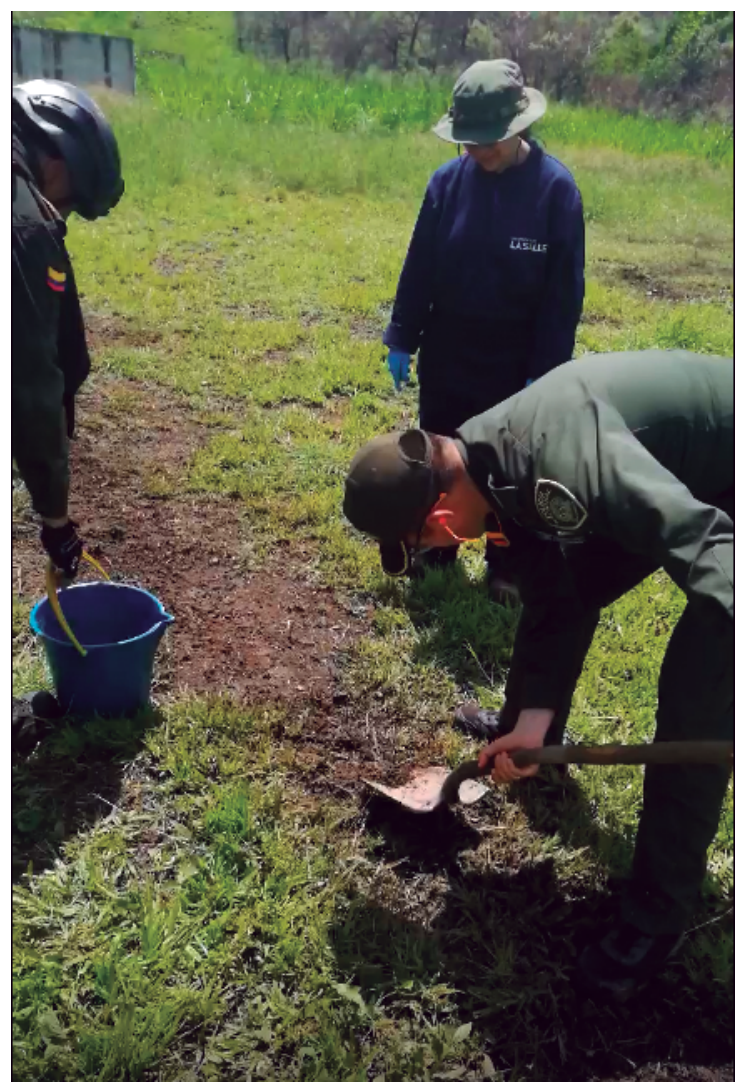

Figura 2. Suelo después de la detonación carga PENT.

Tabla 7. Cantidad de materia orgánica en suelo natural y en suelo expuesto a detonación PENT

\begin{tabular}{cccc} 
Muestra de suelo & $\begin{array}{c}\text { Materia } \\
\text { orgánica (\%) }\end{array}$ & $\begin{array}{c}\text { Diferencia } \\
(\%)\end{array}$ & $\begin{array}{c}\text { Diferencia } \\
\text { porcentual }\end{array}$ \\
\hline Natural & 2,24 & 2,19 & 99,77 \\
\hline Detonado con PENT & 4,43 & & \\
\hline
\end{tabular}

los nitratos tienden a aumentar su concentración en el terreno debido, en primera medida, a la forma en que el explosivo se deposita en el suelo, ya que está sobre este en forma de cordón detonante en toda la superficie aplicada y este cordón está relleno de dicho material explosivo. Por otra parte, el suelo se ve afectado por las reacciones ocurridas en la explosión (División Técnica de Armamentos Terrestres, 1984). En comparación con los resultados obtenidos con explosivo C4, la pentrita causa una mayor variabilidad de este nutriente en el suelo, a pesar de utilizar una menor cantidad de explosivo; se infiere que es a causa del efecto rompedor y la forma comprimida del C4, lo cual hace que su densidad sea muy alta con gran cantidad de energía al ser detonado (Pouretedal, Damiri \& Ghaemi, 20I4), causando una combustión completa que evitó el 
aporte de sus componentes. En cambio, la contextura pulverulenta del PENT hizo que fracciones residuales de nitrato que hacían parte del explosivo no reaccionaran y se mezclaron con el suelo (Villate \& Lozano, 2008).

Tabla 8. Cantidad de nitratos en suelo natural y en suelo expuesto a detonación PENT

\begin{tabular}{lccc} 
Muestra de suelo & $\begin{array}{c}\text { Nitratos } \\
\mathbf{m g} / \mathbf{L}\end{array}$ & $\begin{array}{c}\text { Diferencia } \\
\mathbf{m g} / \mathbf{L}\end{array}$ & Porcentaje \\
\hline Natural & 0,2 & & \\
\hline Detonado con PENT & 2,6 & 2,4 & 1200 \\
\hline
\end{tabular}

La cantidad de $\mathrm{N}$ amoniacal en la muestra de suelo que fue expuesta a la detonación del explosivo PENT se incrementó $140 \%$ en comparación con la muestra de blanco o suelo natural (véase la tabla 9) por las mismas razones expuestas que para los nitratos, en razón a que este tipo de compuestos es familia de nitrogenada y en presencia de agua genera el amonio del nitrógeno amoniacal (División Técnica de Armamentos Terrestres, 1984).

Tabla 9. Cantidad de $\mathrm{N}$ amoniacal en suelo natural y en suelo expuesto a detonación PENT

\begin{tabular}{lccc}
\hline Muestra de suelo & $\begin{array}{c}\text { N/amoniacal } \\
\text { mg/L }\end{array}$ & $\begin{array}{c}\text { Diferencia } \\
\mathbf{m g} / \mathrm{L}\end{array}$ & $\begin{array}{c}\text { Diferencia } \\
\text { porcentual }\end{array}$ \\
\hline Natural & $\mathrm{I}$ & & \\
Detonado con PENT & 2,4 & $\mathrm{I}, 4$ & 140 \\
\hline
\end{tabular}

\section{Observación en campo luego de}

la detonación de pentolita

En las detonaciones se utilizaron cargas de $125 \mathrm{~g}$ y $250 \mathrm{~g}$ de explosivo pentolita. Ambas cargas generaron daño visible causando fractura en la superficie de suelo; asimismo, se produjo una expulsión brusca de nubes oscuras en el ambiente por la combustión del material orgánico. Con este tipo de explosivo se evidencia un mayor daño en la primera capa del suelo (horizonte 0 ) en comparación con los dos primeros explosivos.

Al ser detonada la carga de $125 \mathrm{~g}$ produce una nube de humo color gris claro de, aproximadamente, $5 \mathrm{~m}$ de alto, la cual se fue dispersando por un tiempo estimado de 45 $s$ hasta desaparecer; la afectación que produjo en el terreno fue de $4 \mathrm{~m}$ de ancho $\times 5 \mathrm{~m}$ largo y $\mathrm{I} \mathrm{cm}$ profundidad (véase la figura 3 ).

Al ser detonada la carga de $250 \mathrm{~g}$ produce una nube de humor color gris claro de aproximadamente $7 \mathrm{~m}$ de altura, la cual se fue dispersando por un tiempo estimado de I min hasta desaparecer; la afectación que produjo en el terreno fue de $5 \mathrm{~m}$ de ancho $\times 7 \mathrm{~m}$ de largo y $2 \mathrm{~cm}$ de profundidad (véase la figura 4).

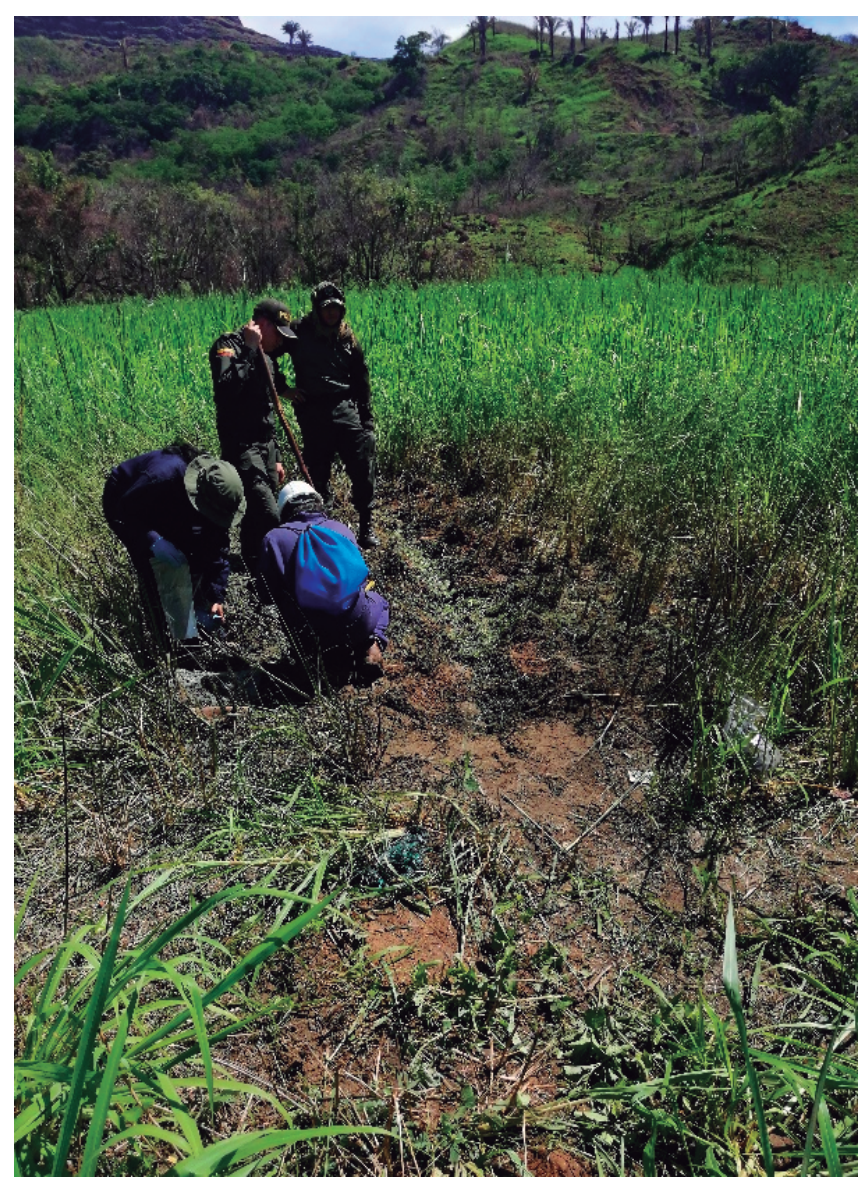

Figura 3. Suelo después de la detonación carga de pentolita de $125 \mathrm{~g}$.

Se observa una reducción de $40,6 \%$ de la materia orgánica para las muestras en las que se utilizó $125 \mathrm{~g}$ de pentolita en comparación con la muestra de blanco (véase la tabla 10). Además, para las muestras en las que se utilizaron cargas de $250 \mathrm{~g}$ de pentolita se observa una reducción en MO del 55,8\% en comparación con la $\mathrm{MO}$ en el suelo natural, debido al desplazamiento del material por la onda explosiva. También se observa mayor afectación para este compuesto en relación con la carga de $125 \mathrm{~g}$, debido a la mayor cantidad de explosivo (véase la tabla 10). Dado que es este el primer trabajo en campo realizado en el país con este tipo de suelo no se tiene punto de comparación.

La muestra que fue sometida a la detonación de $125 \mathrm{~g}$ de pentolita presenta un aumento de nitratos de $400 \%$ en comparación con la cantidad de este nutriente en la muestra inicial (véase la tabla II). Para este existe una variación importante en el indicador debido a las reacciones 


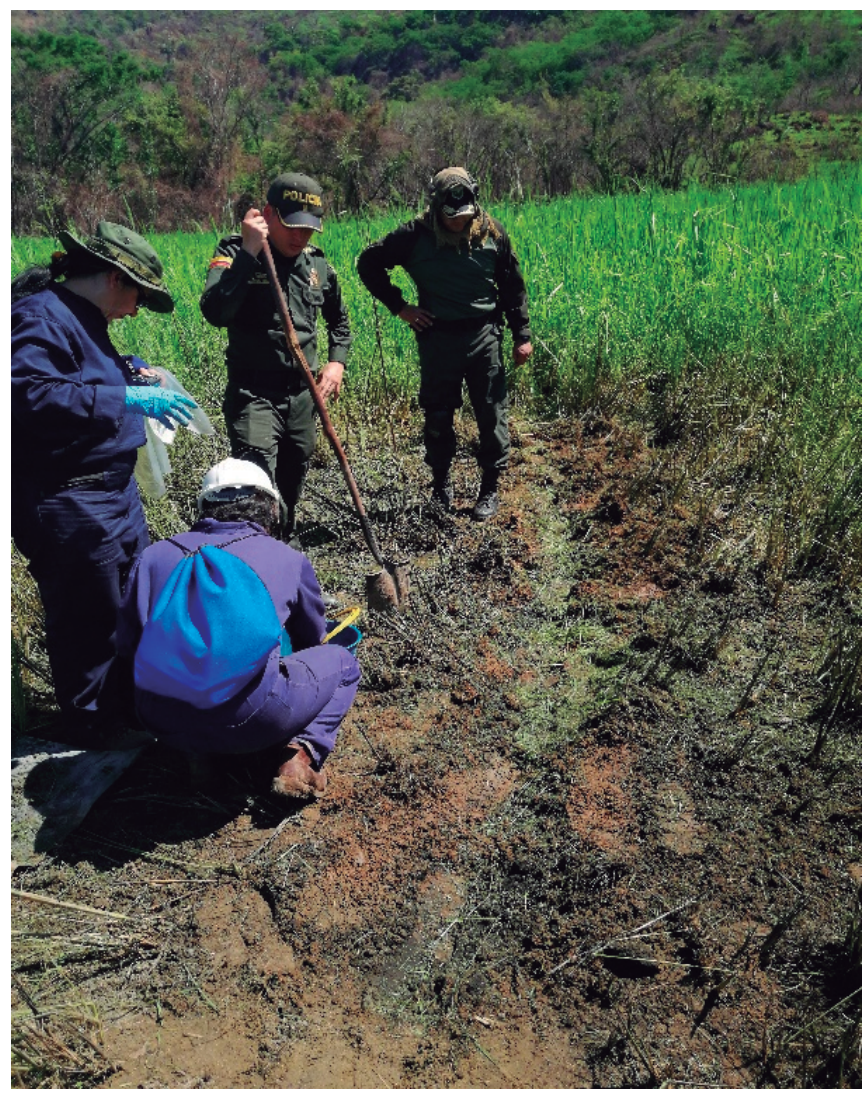

Figura 4. Suelo después de la detonación de la carga de pentolita de $250 \mathrm{~g}$.

Tabla I0. Cantidad de materia orgánica en suelo natural y en suelo expuesto a detonación pentolita

\begin{tabular}{lccc} 
Muestra de suelo & $\begin{array}{c}\text { Materia } \\
\text { orgánica \% }\end{array}$ & $\begin{array}{c}\text { Diferen- } \\
\text { cia \% }\end{array}$ & $\begin{array}{c}\text { Diferencia } \\
\text { porcentual }\end{array}$ \\
\hline $\begin{array}{l}\text { Natural } \\
\text { Detonado con pentolita }\end{array}$ & 1,24 & & \\
$\begin{array}{l}\text { (I } 25 \text { g) } \\
\begin{array}{l}\text { Detonado con pentolita } \\
(250 \text { g) }\end{array}\end{array}$ & 0,99 & 0,91 & 40,6 \\
\hline
\end{tabular}

químicas de descomposición del material (División Técnica de Armamentos Terrestres, 1984).

Al utilizar las cargas de $250 \mathrm{~g}$ se evidencia la mayor variación de presencia de nitratos en las muestras, y se observa un aumento del $7550 \%$ en comparación con el blanco (véase la tabla II). Se infiere que la variación del contenido de nitrato en el suelo depende de la cantidad de explosivo pentolita que se utilice y de la reacción química presentada (División Técnica de Armamentos Terrestres, 1984). Cabe anotar que los niveles de variación en el índice de nitrato encontrado en la muestra de suelo expuesta a la detonación de la pentolita I (125 g) no sobrepasan la cantidad aceptada por la Organización de las Naciones Unidas para la Agricultura y la Alimentación (FAO/OMS) de 0 a $3,7 \mathrm{mg} / \mathrm{kg}$; sin embargo, la muestra de suelo expuesta a la detonación de pentolita 2 ( $250 \mathrm{~g}$ ) supera en un $408 \%$ (véase la tabla II) la cantidad máxima aceptada (Antón \& Lizaso, 200I).

Tabla II. Cantidad de nitratos en suelo natural y en suelo expuesto a detonación pentolita

\begin{tabular}{lccc}
\multicolumn{1}{|c}{ Muestra de suelo } & $\begin{array}{c}\text { Nitratos } \\
\mathrm{mg} / \mathrm{L}\end{array}$ & $\begin{array}{c}\text { Diferencia } \\
\mathrm{mg} / \mathrm{L}\end{array}$ & $\begin{array}{c}\text { Diferencia } \\
\text { porcentual }\end{array}$ \\
\hline Natural & 0,2 & & \\
$\begin{array}{l}\text { Detonado con pentolita } \\
(125 \mathrm{~g})\end{array}$ & $\mathrm{I}$ & 0,8 & 400 \\
$\begin{array}{l}\text { Detonado con pentolita } \\
(250 \mathrm{~g})\end{array}$ & 15,3 & 15,1 & 7550 \\
\hline
\end{tabular}

La cantidad de $\mathrm{N}$ amoniacal en la muestra que fue expuesta a la detonación de la carga explosiva de pentolita 125 g aumentó $200 \%$ en comparación con la muestra de suelo natural (véase la tabla 12).Además, la de la muestra que fue expuesta a la detonación de la carga explosiva de pentolita $250 \mathrm{~g}$ aumentó en un 190\% en comparación con la muestra de suelo natural (véase la tabla 12). Estas variaciones reflejan una reacción de los compuestos nitrados con el vapor de agua generado en la reacción (División Técnica de Armamentos Terrestres, 1984). Estos residuos pueden afectar las fuentes hídricas cercanas por filtración, así como generar eutrofización (González \& Murillo, 2016); esto es un factor de riesgo si se tiene en cuenta que concentraciones altas de estos componentes afectan la salud animal y humana (Vega, 2017).

Tabla I2. Cantidad de $\mathrm{N}$ amoniacal en suelo natural y en suelo expuesto a detonación pentolita

\begin{tabular}{lccc}
\hline \multicolumn{1}{|c}{ Muestra de suelo } & $\begin{array}{c}\text { N amoniacal } \\
\mathbf{m g} / \mathbf{L}\end{array}$ & $\begin{array}{c}\text { Diferencia } \\
\mathbf{m g} / \mathbf{L}\end{array}$ & $\begin{array}{c}\text { Diferencia } \\
\text { porcentual }\end{array}$ \\
\hline Natural & $\mathrm{I}$ & & \\
$\begin{array}{l}\text { Detonado con pentolita } \\
(125 \mathrm{~g})\end{array}$ & 3 & 2 & 200 \\
$\begin{array}{l}\text { Detonado con pentolita } \\
(250 \mathrm{~g})\end{array}$ & 2,9 & 1,9 & 190 \\
\hline
\end{tabular}

\section{Conclusiones}

Se puede evidenciar que la detonación de los explosivos $\mathrm{C} 4$ y pentolita en el suelo provocaron cambios en la composición fisicoquímica de estos, en particular una reducción de la materia orgánica debida a que la onda explosiva puede llegar a remover toda la capa superficial del suelo por desplazamiento en proporciones que dependen de la forma y la cantidad en las que se ubica el material 
explosivo en el suelo. Este impacto ambiental negativo contribuye a la erosión que causa la pérdida de una capa de 0,5 a $2 \mathrm{~cm}$ del horizonte más productivo.

La detonación con pentrita en el suelo fue el único material químico explosivo que provocó un aumento de la materia orgánica debido a la forma en que fue acondicionado en terreno (cordón detonante) y a su estructura alifática con alto contenido de carbonos, de modo que dejó estos residuos en suelo.

Por otra parte, el suelo sometido a las detonaciones de material explosivo en comparación con la muestra de suelo natural presentó mayores concentraciones de nitratos y nitrógeno amoniacal en razón a que los explosivos aportan estos componentes al suelo, pues estos se encuentran contenidos en su estructura; si el contacto es directo con el suelo las concentraciones serán mayores. Cabe tener en cuenta que los valores de estas especies son mayores a las reportadas para actividades agrícolas, lo que implica problemas posteriores de eutroficación y pérdida de salud del suelo.

Si se tienen en cuenta los resultados obtenidos en la investigación, se concluye que el explosivo que causó mayor impacto en el suelo objeto de estudio fue el expuesto a las detonaciones con $\mathrm{C} 4$, del cual se obtuvieron los valores más altos en variación de materia orgánica (véase la tabla 4) y $\mathrm{N}$ amoniacal (véase la tabla 6); se infiere que esto puede ser debido al efecto rompedor y la forma comprimida del $\mathrm{C} 4$ que lo hace el explosivo de mayor poder de destrucción. Además, es importante anotar que el grado de afectación ambiental está determinado por la cantidad de material explosivo, el tipo, la presentación física del explosivo y la ubicación de este en el suelo, ya que los valores observados en la investigación evidenciaron esta dependencia.

\section{Recomendaciones y consideraciones}

Dada la afectación ambiental presentada en el recurso suelo en el presente estudio y con base en las investigaciones desarrolladas en conjunto con la Universidad de La Salle, se recomienda no realizar detonaciones ni destrucciones de sustancia explosiva en suelos determinados para la actividad agrícola, teniendo en cuenta el deterioro observado, principalmente, en la materia orgánica.
Es necesario diseñar y estandarizar formalmente un protocolo de actuación en terreno dirigido a las autoridades ambientales que permita la determinación del impacto ambiental asociado a suelos, y mantener así las medidas técnicas y de bioseguridad para el desarrollo del procedimiento en la recolección de información.

Se considera de vital importancia que la fuerza pública continúe con los controles operativos de incautación de sustancias explosivas con la finalidad de prevenir acciones que atenten contra la integridad de las personas, así como prevenir el impacto ambiental que se puede generar al detonar dichas sustancias en el medio ambiente.

Finalmente, se recomienda a los científicos especializados continuar con el desarrollo de nuevos estudios en la materia que permitan la generación de nuevos conocimientos acerca de la contaminación ambiental y sus consecuencias para el ambiente.

\section{Agradecimientos}

A Dios, en primer lugar, así como nuestro sincero agradecimiento a la Escuela de Investigación Criminal de la Policía Nacional de Colombia y a la Universidad de La Salle por brindar los conocimientos, los medios, las herramientas y los espacios propicios para el desarrollo de la presente investigación.

\section{Referencias}

Antón, A., \& Lizaso, J. (200I). Nitratos, nitritos y nitrosaminas. Fundación Ibérica para la Seguridad Alimentaria.

División Técnica de Armamentos Terrestres. (1984). Manual Técnico de Explosivos. Dirección General de Armamento y Material. Ministerio de Defensa. España.

García, A. (2013). Degradación del pentaeritritol tetranitrato (PENT). Universidad de La Sabana.

González, R. \& Murillo, J. (2016). Metodología para la determinación de impactos ambientales en los suelos desminados con fines agrícolas. Ediciones Unisalle. https://ciencia.lasalle.edu. co/ing_ambiental_sanitaria/400/

González, R. \& Ortiz, B. (2020). Propuesta normativa ambiental en el marco del desminado humanitario para la utilización del suelo con fines agropecuarios. Ediciones Unisalle. https://doi. org/I 0.19052/978-958-5 |36-08-3 
González, R., Ortiz, B., \& Erazo, M. (2017). Impacto ambiental en los suelos afectados por la presencia de artefactos explosivos. Revista Científica Monfragüe Resiliente, 9(I), 14-25.

Hach Company. (200I). Manual de análisis de agua. Procedimientos seleccionados del manual de Hach sobre análisis de agua. Loveland.

Hernández, R., Fernández, C., \& Baptista, P. (2010). Metodología de la investigación ( $4^{\mathrm{a}}$ ed.). McGraw-Hill Interamericana.

Hernández, R., Fernández, C., \& Baptista, P. (2014). Metodología de la investigación ( $6^{\mathrm{a}}$ ed.). McGraw-Hill.

Instituto Geográfico Agustín Codazzi. (1997). Estudio general de suelos del departamento del Tolima. http://metadatos. igac.gov.co/geonetwork/srv/spa/catalog.search\#/metadata/e2 I 3fa32-53 I c-4ac2-ad45-68d0e727| 2c |

Instituto Geográfico Agustín Codazzi. (2017). Muestreo de suelos. https://www.igac.gov.co/sites/igac.gov.co/files/guiademuestreo.pdf

Okalebo, J. R., Gathua, K.W., \& Woomer, P. L. (2002). Laboratory methods of soil and plant analysis: a working manual ( $2^{\mathrm{a}}$ ed.). Sacred Africa.
Pouretedal, H., Damiri, S., \& Ghaemi, E. (20I4). Non-isothermal studies on the thermal decomposition of $\mathrm{C} 4$ explosive using the TG/DTA technique. Central European Journal of Energetic Materials, I I (3), 4I5-4I6.

Programa de las Naciones Unidas para el Desarrollo. (2020). Objetivos de desarrollo sostenible. https://www.undp.org/content/undp/es/home/sustainable-development-goals.html

Rojas, J. J. (2015). Fertilidad de suelos en plantaciones forestales del trópico colombiano. Universidad Nacional de Colombia, Sede Medellín.

Teddlie, C., \& Tashakkori,A. (2003). Handbook of mixed methods in social \& behavioral research. Sage Publications.

Vega, O. C. (2017). Problemas ambientales y de salud derivados del uso de fertilizantes nitrogenados. Universidad Complutense de Madrid.

Villate, C., \& Lozano, J. (2008). Evaluación de la afectación ambiental de suelos a partir de las variaciones fisicoquímicas generadas por explosiones de anfo y pólvora (tipo barragán) en suelos arcillosos de los municipios de Cogua y Nemocón. Universidad de La Salle. https://ciencia.lasalle.edu.co/ing_ambiental_sanitaria/592 